ELECTRONIC RESEARCH ANNOUNCEMENTS OF THE AMERICAN MATHEMATICAL SOCIETY

Volume 11, Pages 78-88 (September 28, 2005)

S $1079-6762(05) 00150-2$

\title{
A PROOF OF A GENERALIZATION OF DELIGNE'S CONJECTURE
}

\author{
YAKOV VARSHAVSKY
}

(Communicated by Svetlana Katok)

\begin{abstract}
The goal of this paper is to give a simple proof of Deligne's conjecture on the Lefschetz trace formula (proven by Fujiwara) and to generalize it to the situation appearing in the forthcoming joint paper with D. Kazhdan. Our proof holds in the realm of ordinary algebraic geometry and does not use rigid geometry.
\end{abstract}

\section{INTRODUCTION}

Suppose we are given a correspondence $X \stackrel{c_{1}}{\longleftarrow} C \stackrel{c_{2}}{\longrightarrow} X$ of separated schemes of finite type over a separably closed field $k$, an " $\ell$-adic sheaf" $\mathcal{F} \in D_{c}^{b}\left(X, \overline{\mathbb{Q}_{l}}\right)$, and a morphism $u: c_{2 !} c_{1}^{*} \mathcal{F} \rightarrow \mathcal{F}$. If $c_{1}$ is proper, then $u$ gives rise to an endomorphism $R \Gamma_{c}(u): R \Gamma_{c}(X, \mathcal{F}) \rightarrow R \Gamma_{c}(X, \mathcal{F})$.

When $X$ is proper, the general Lefschetz-Verdier trace formula [II, Cor. 4.7] asserts that the trace $\operatorname{Tr}\left(R \Gamma_{c}(u)\right)$ equals the sum $\sum_{\beta \in \pi_{0}(F i x(c))} L T_{\beta}(u)$, where $F i x(c):=\left\{y \in C \mid c_{1}(y)=c_{2}(y)\right\}$ is the scheme of fixed points of $c$, and $L T_{\beta}(u)$ is a so-called "local term" of $u$ at $\beta$. This result has two drawbacks: it fails when $X$ is not proper, and the "local terms" are very inexplicit.

Deligne conjectured that the situation becomes better if $X \stackrel{c_{1}}{\longleftarrow} C \stackrel{c_{2}}{\longrightarrow} X$ is defined over a finite field $\mathbb{F}_{q}, c_{2}$ is quasi-finite, and we twist $c_{1}$ by a sufficiently high power of the geometric Frobenius morphism. More precisely, he conjectured that in this case the Lefschetz-Verdier trace formula holds also for a non-proper $X$ 's, Fix $(c)$ is finite, and for each $y \in c_{1}^{-1}(x) \cap c_{2}^{-1}(x)$, the local term $L T_{y}(u)$ equals the trace of the endomorphism $u_{y}: \mathcal{F}_{x} \rightarrow \mathcal{F}_{x}$, induced by $u$.

The conjecture was first proven by Pink $\mathrm{Pi}$ assuming the resolution of singularities, and then by Fujiwara $[\mathrm{Fu}$ ] unconditionally.

The theorem of Fujiwara has a fundamental importance for Langlands' program. For example, it was crucially used by Flicker-Kazhdan, Harris-Taylor, and Lafforgue.

In a joint project $[\mathrm{KV}$ with David Kazhdan on the global Langlands correspondence over function fields we needed a generalization of Deligne's conjecture. Namely, instead of assuming that $c_{1}$ is proper we assumed that there exists an open subset $U \subset X$ such that $\left.c_{1}\right|_{c_{1}^{-1}(U)}$ is proper, $X \backslash U$ is "locally $c$-invariant", and

Received by the editors May 16, 2005.

2000 Mathematics Subject Classification. Primary 14F20; Secondary 11G25, 14 G15.

Key words and phrases. Lefschetz trace formula, Deligne's conjecture.

The work was supported by the Israel Science Foundation (Grant No. 555/04).

(C)2005 American Mathematical Society 
$\mathcal{F}$ vanishes on $X \backslash U$. In this case, $u$ still gives rise to an endomorphism $R \Gamma_{c}(u)$, and the main result of the present work asserts that the conclusion of Deligne's conjecture holds in this case.

The strategy of our proof is similar to that of $[\mathrm{Pi}]$ and $[\mathrm{Fu}]$ : first we reduce the problem to vanishing of local terms $L T_{\beta}$, then we make the correspondence "contracting" by twisting it with a sufficiently high power of Frobenius, and finally we show the vanishing of local terms for "contracting" correspondences.

Our approach differs from that of Fujiwara in two respects. First of all, our notion of a "contracting" correspondence is much simpler both to define and to use. Namely, we use the most naive notion of an "infinitesimally" contracting correspondence, which has a simple geometric description in terms of a "deformation to the normal cone". As a result, our bound on the power of Frobenius is sharper and more explicit.

Secondly, to prove a generalization of Deligne's conjecture described above, we work "locally". More precisely, as in [Pi], to show the vanishing of "local terms", we first show the vanishing of the so-called "trace maps", from which "local terms" are obtained by integration.

Finally we would like to emphasize that our result is ultimately an assertion about the geometry of correspondences and not about sheaves. Also our proof applies without any changes to more general situations like compactifiable algebraic spaces or Deligne-Mumford stacks.

\section{NOTATION AND CONVENTIONS}

Throughout the paper, all schemes will be separated schemes of finite type over a separably closed field $k$. We fix a prime $l$, invertible in $k$, and a commutative ring with identity $\Lambda$, which is either finite and is annihilated by some power of $l$, or a finite extension of $\mathbb{Z}_{l}$ or $\mathbb{Q}_{l}$.

To each scheme $X$ as above, we associate a derived category $D_{c t f}^{b}(X, \Lambda)$ of complexes of $\Lambda$-modules of finite tor-dimension with constructible cohomology. This category is known to be stable under the six operations $f^{*}, f^{!}, f_{*}, f_{!}, \otimes$ and $\mathcal{R}$ Hom.

For each $X$ as above, we denote by $\pi_{X}: X \rightarrow$ Spec $k$ the structure morphism, by $K_{X}=\pi_{X}^{!} \Lambda$ the dualizing complex of $X$, and by $\mathbb{D}=\mathcal{R} \operatorname{Hom}\left(\cdot, K_{X}\right)$ the Verdier duality functor. We also write $R \Gamma_{c}(X, \cdot)$ instead of $\pi_{X !}$. For an embedding $f$ : $Y \hookrightarrow X$, we will write $\left.\cdot\right|_{Y}$ instead of $f^{*}$.

For a closed subscheme $Z \subset X$, we denote by $\mathcal{I}_{Z} \subset \mathcal{O}_{X}$ the sheaf of ideals of $Z$. For a morphism $f: X^{\prime} \rightarrow X$, we denote by $f^{-1}(Z)$ the schematic inverse image of $Z$, i.e., the closed subscheme of $X^{\prime}$ such that $\mathcal{I}_{f^{-1}(Z)}=f^{\cdot}\left(\mathcal{I}_{Z}\right) \cdot \mathcal{O}_{X^{\prime}} \subset \mathcal{O}_{X^{\prime}}$, where $f^{\prime}$ ' is the pullback map for functions. We will also identify a closed subset of $X$ with the corresponding closed reduced subscheme.

Let $\overline{\mathbb{F}_{q}}$ be an algebraic closure of the finite field $\mathbb{F}_{q}$. We say that an object $\mathcal{X}$ over $\overline{\mathbb{F}_{q}}$ is defined over $\mathbb{F}_{q}$ if it is a pullback of the corresponding object over $\mathbb{F}_{q}$.

\section{ACKNOWLEDGMENTS}

This work would not be possible without David Kazhdan, who among other things explained to me how to define $R \Gamma_{c}(u)$ in the case described above and suggested that an analog of Deligne's conjecture should work in this situation.

Also the author thanks Alexander Beilinson, who gave a reference to $\mathrm{Ve}$ and whose comments and suggestions helped to simplify and improve the exposition. 
Part of the work was done while the author visited the University of Chicago and Northwestern University. The author thanks these institutions for hospitality and financial support.

\section{Formulation of the Result}

\subsection{Preliminaries on correspondences.}

1.1.1. Definition. By a correspondence, we mean a morphism of schemes of the form $c=\left(c_{1}, c_{2}\right): C \rightarrow X_{1} \times X_{2}$. By a c-morphism, we mean a morphism $u$ : $c_{2 !} c_{1}^{*} \mathcal{F}_{1} \rightarrow \mathcal{F}_{2}$ for some $\mathcal{F}_{1} \in D_{c t f}^{b}\left(X_{1}, \Lambda\right)$ and $\mathcal{F}_{2} \in D_{c t f}^{b}\left(X_{2}, \Lambda\right)$.

1.1.2. Remark. A c-morphism is usually called a cohomological correspondence lifting $c$.

1.1.3. Restriction of correspondences. Let $c$ and $u$ be as in 1.1.1 and $C^{0} \subset C$, $X_{1}^{0} \subset X_{1}$ and $X_{2}^{0} \subset X_{2}$ open subschemes (resp. closed subschemes satisfying $\left.c_{2}^{-1}\left(X_{2}^{0}\right)_{\text {red }}=C_{\text {red }}^{0}\right)$ such that $c$ induces a correspondence $c^{0}: C^{0} \rightarrow X_{1}^{0} \times X_{2}^{0}$.

Then we have a base change morphism $B C:\left.c_{2 !}^{0}\left(\left.\mathcal{F}\right|_{C^{0}}\right) \rightarrow\left(c_{2 !} \mathcal{F}\right)\right|_{X_{2}^{0}}$ for every $\mathcal{F} \in D_{\text {ctf }}^{b}(C, \Lambda)$, hence $u$ restricts to a $c^{0}$-morphism

$$
u^{0}: c_{2 !}^{0} c_{1}^{0 *}\left(\left.\mathcal{F}_{1}\right|_{X_{1}^{0}}\right)=\left.\left.c_{2 !}^{0}\left(\left.c_{1}^{*} \mathcal{F}_{1}\right|_{C^{0}}\right) \stackrel{B C}{\longrightarrow}\left(c_{2 !} c_{1}^{*} \mathcal{F}_{1}\right)\right|_{X_{2}^{0}} \stackrel{u}{\longrightarrow} \mathcal{F}_{2}\right|_{X_{2}^{0}} .
$$

1.1.4. Endomorphism on the cohomology. Let $c$ and $u$ be as in 1.1.1.

a) Assume that $c_{1}$ is proper. Then $u$ induces a morphism

$$
R \Gamma_{c}(u): R \Gamma_{c}\left(X_{1}, \mathcal{F}_{1}\right) \stackrel{c_{1}^{*}}{\longrightarrow} R \Gamma_{c}\left(C, c_{1}^{*} \mathcal{F}_{1}\right)=R \Gamma_{c}\left(X_{2}, c_{2 !} c_{1}^{*} \mathcal{F}_{1}\right) \stackrel{u}{\longrightarrow} R \Gamma_{c}\left(X_{2}, \mathcal{F}_{2}\right) \text {. }
$$

b) More generally, assume that there exists an open subset $U_{1} \subset X_{1}$ such that $\left.c_{1}\right|_{c_{1}^{-1}\left(U_{1}\right)}: c_{1}^{-1}\left(U_{1}\right) \rightarrow U_{1}$ is proper and $\left.\mathcal{F}_{1}\right|_{X_{1} \backslash U_{1}}=0$. Let $c^{0}: c_{1}^{-1}\left(U_{1}\right) \rightarrow U_{1} \times X_{2}$ be the restriction of $c$, and $u^{0}$ the restriction of $u$ to $c^{0}$ (see 1.1.3). Then $c_{1}^{0}$ is proper, hence by a) $u^{0}$ gives rise to a morphism $R \Gamma_{c}\left(u^{0}\right): R \Gamma_{c}\left(U_{1},\left.\mathcal{F}_{1}\right|_{U_{1}}\right) \rightarrow R \Gamma_{c}\left(X_{2}, \mathcal{F}_{2}\right)$.

On the other hand, the canonical map $j_{!}: R \Gamma_{c}\left(U_{1},\left.\mathcal{F}_{1}\right|_{U_{1}}\right) \rightarrow R \Gamma_{c}\left(X_{1}, \mathcal{F}_{1}\right)$ is an isomorphism, and therefore $u$ gives rise to a morphism

$$
R \Gamma_{c}(u):=R \Gamma_{c}\left(u^{0}\right) \circ\left(j_{!}\right)^{-1}: R \Gamma_{c}\left(X_{1}, \mathcal{F}_{1}\right) \rightarrow R \Gamma_{c}\left(X_{2}, \mathcal{F}_{2}\right) .
$$

1.1.5. Twisting of correspondences. For a scheme $X / \overline{\mathbb{F}_{q}}$, which defined over $\mathbb{F}_{q}$, we denote by $\operatorname{Fr}_{X}=\operatorname{Fr}_{X, q}: X \rightarrow X$ the geometric Frobenius morphism. For a correspondence $c: C \rightarrow X_{1} \times X_{2}$ defined over $\mathbb{F}_{q}$ and an integer $n \in \mathbb{N}$, we denote by $c^{(n)}$ the correspondence $\left(c_{1}^{(n)}, c_{2}\right): C \rightarrow X_{1} \times X_{2}$, where $c_{1}^{(n)}=\operatorname{Fr}_{X_{1}}^{n} \circ c_{1}=c_{1} \circ \operatorname{Fr}_{C}^{n}$.

1.1.6. Notation. For a correspondence $c: C \rightarrow X \times X$, let $\Delta$ be the diagonal map $X \hookrightarrow X \times X$, put Fix $(c):=c^{-1}(\Delta(X)) \subset C$, and denote by $\Delta^{\prime}:$ Fix $(c) \hookrightarrow C$ and $c^{\prime}:$ Fix $(c) \rightarrow X$ the inclusion map and the restriction of $c$, respectively. We call Fix $(c)$ the scheme of fixed points of $c$.

1.1.7. Quasi-finite case. Let $c: C \rightarrow X \times X$ be a correspondence such that $c_{2}$ is quasi-finite, and let $u: c_{2 !} c_{1}^{*} \mathcal{F} \rightarrow \mathcal{F}$ be a $c$-morphism.

Each fiber $\left(c_{2 !} c_{1}^{*} \mathcal{F}\right)_{x}$ decomposes as $\bigoplus_{y \in c_{2}^{-1}(x)}\left(c_{1}^{*} \mathcal{F}\right)_{y}=\bigoplus_{y \in c_{2}^{-1}(x)} \mathcal{F}_{c_{1}(y)}$. Hence for each $y \in c_{2}^{-1}(x)$, the map $u$ induces a morphism $u_{y}:=\left.u_{x}\right|_{\mathcal{F}_{c_{1}(y)}}: \mathcal{F}_{c_{1}(y)} \rightarrow \mathcal{F}_{x}$. In particular, for each $y \in c_{2}^{-1}(x) \cap c_{1}^{-1}(x)$, we get an endomorphism $u_{y}: \mathcal{F}_{x} \rightarrow \mathcal{F}_{x}$. 
1.1.8. Definition. Let $c: C \rightarrow X \times X$ be a correspondence. We say that a closed subset $Z \subset X$ is locally c-invariant if for each $x \in Z$ there exists an open neighborhood $U \subset X$ of $x$ such that $c_{1}\left(c_{2}^{-1}(Z \cap U)\right)$ is set-theoretically contained in $Z \cup(X \backslash U)$.

\subsection{Main theorem.}

1.2.1. Notation. Let $f: Y \rightarrow X$ be a morphism of Noetherian schemes.

a) For a closed reduced subscheme $Z \subset X$, we denote by $\operatorname{ram}(f, Z)$ (the ramification of $f$ at $Z$ ) the smallest positive integer $m$ such that $\left(\mathcal{I}_{\left(f^{-1}(Z)\right)_{\text {red }}}\right)^{m} \subset \mathcal{I}_{f^{-1}(Z)}$.

b) If $f$ is quasi-finite, we denote by $\operatorname{ram}(f)$ (the ramification degree of $f$ ) the maximum of $\operatorname{ram}(f, x)$, where $x$ runs over the set of all closed points of $X$.

Now we are ready to formulate our main result.

1.2.2. Theorem. Let $c: C \rightarrow X \times X$ be a correspondence defined over $\mathbb{F}_{q}$.

a) Assume that $c_{2}$ is quasi-finite. Then for every $n \in \mathbb{N}$ with $q^{n}>\operatorname{ram}\left(c_{2}\right)$, the set Fix $\left(c^{(n)}\right)$ is finite.

b) Let $U \subset X$ be an open subset defined over $\mathbb{F}_{q}$ such that $\left.c_{1}\right|_{c_{1}^{-1}(U)}$ is proper, $\left.c_{2}\right|_{c_{2}^{-1}(U)}$ is quasi-finite, and $X \backslash U$ is locally c-invariant.

Then there exists a positive integer $d \geq \operatorname{ram}\left(\left.c_{2}\right|_{c_{2}^{-1}(U)}\right)$ with the following property: For every $\mathcal{F} \in D_{\text {ctf }}^{b}(X, \Lambda)$ with $\left.\mathcal{F}\right|_{X \backslash U}=0$, every $n \in \mathbb{N}$ with $q^{n}>d$ and every $c^{(n)}$-morphism $u: c_{2 !}\left(c_{1}^{(n)}\right)^{*} \mathcal{F} \rightarrow \mathcal{F}$, we have an equality

$$
\operatorname{Tr}\left(R \Gamma_{c}(u)\right)=\sum_{y \in F i x\left(c^{(n)}\right) \cap c^{\prime-1}(U)} \operatorname{Tr}\left(u_{y}\right) .
$$

c) In the notation of $b)$, assume that $X$ and $C$ are proper. Then $d:=\max \left\{\operatorname{ram}\left(\left.c_{2}\right|_{c_{2}^{-1}(U)}\right), \operatorname{ram}\left(c_{2}, X \backslash U\right)\right\}$ satisfies the conclusion of $\left.b\right)$.

1.2.3. Remark. a) Note that both sides of (1.1) are well defined. Namely, $R \Gamma_{c}(u)$ was defined in 1.1.4 b), $u_{y}$ was defined in 1.1.7, and the sum is finite by a).

b) In the notation of $1.2 .2 \mathrm{~b})$, assume that $\mathcal{F} \in D_{\text {ctf }}^{b}(X, \Lambda)$ is equipped with a morphism $\psi: \operatorname{Fr}_{X}^{*} \mathcal{F} \rightarrow \mathcal{F}$ (say, $\mathcal{F}$ is a Weil sheaf) and with a $c$-morphism $u: c_{2 !} c_{1}^{*} \mathcal{F} \rightarrow \mathcal{F}$. Then for each $n \in \mathbb{N}, \mathcal{F}$ is equipped with a $c^{(n)}$-morphism $u^{(n)}:=u \circ \psi^{n}: c_{2 !}\left(c_{1}^{(n)}\right)^{*} \mathcal{F} \rightarrow \mathcal{F}$, so one can apply formula (1.1). In the case $U=X$, the assertion thus reduces to Deligne's conjecture proven by Fujiwara $\mathrm{Fu}$.

c) The constant $d$ in $1.2 .2 \mathrm{~b}$ ) can be made explicit. Namely, one can see from the proof that the picture can be compactified, and then $1.2 .2 \mathrm{c}$ ) gives an estimate for $d$.

\section{Proof of the MAIN THEOREM}

\subsection{Push-forward of cohomological correspondences.}

2.1.1. Definition. By a morphism from a correspondence $c: C \rightarrow X_{1} \times X_{2}$ to a correspondence $b: B \rightarrow Y_{1} \times Y_{2}$ we mean a triple $[f]=\left(f_{1}, f^{\natural}, f_{2}\right)$ making the following diagram commutative:

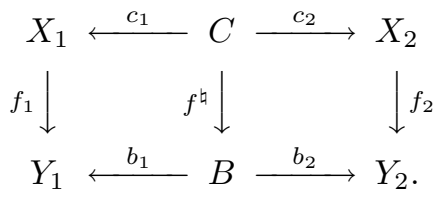


2.1.2. Construction. In the notation of 2.1.1 assume that either

(i) the left-hand square of (2.1) is Cartesian, or

(ii) the morphisms $f_{1}$ and $f^{\natural}$ are proper, or

(iii) the morphisms $c_{1}$ and $b_{1}$ are proper.

In all these cases, we have base change morphisms $B C: b_{1}^{*} f_{1 !} \rightarrow f_{!}^{\natural} c_{1}^{*}$. Hence every $c$-morphism $u: c_{2} ! c_{1}^{*} \mathcal{F}_{1} \rightarrow \mathcal{F}_{2}$ gives rise to a $b$-morphism

$$
[f]_{!}(u): b_{2 !} b_{1}^{*}\left(f_{1 !} \mathcal{F}_{1}\right) \stackrel{B C}{\longrightarrow} b_{2 !} f_{!}^{\natural} c_{1}^{*} \mathcal{F}_{1}=f_{2 !} c_{2 !} c_{1}^{*} \mathcal{F}_{1} \stackrel{u}{\longrightarrow} f_{2 !} \mathcal{F}_{2} .
$$

2.1.3. Example. For each correspondence $c$, there is a structure morphism $[\pi]_{c}$ from $c$ to the trivial correspondence $c_{t r}: \operatorname{Spec} k \rightarrow \operatorname{Spec} k \times \operatorname{Spec} k$. Moreover, if $c_{1}$ is proper, then $[\pi]_{c}$ satisfies assumption (iii) of $\left[2.1 .2\right.$, and $[\pi]_{c !}$ coincides with the map $R \Gamma_{c}$, defined in $1.1 .4 \mathrm{a}$ ).

\subsection{Trace maps and local terms.}

2.2.1. Definition of trace maps. Let $c: C \rightarrow X \times X$ be a correspondence.

a) Denote by $\underline{\mathcal{T} r}: \mathcal{R} \operatorname{Hom}\left(c_{1}^{*} \mathcal{F}, c_{2}^{!} \mathcal{F}\right) \rightarrow \Delta_{*}^{\prime} K_{F i x(c)}$ the composition

$$
\mathcal{R} H \operatorname{Hom}\left(c_{1}^{*} \mathcal{F}, c_{2}^{!} \mathcal{F}\right) \stackrel{\sim}{\rightarrow} c^{!}(\mathbb{D} \mathcal{F} \otimes \mathcal{F}) \rightarrow c^{!} \Delta_{*} K_{X} \stackrel{\sim}{\rightarrow} \Delta_{*}^{\prime} c^{\prime !} K_{X}=\Delta_{*}^{\prime} K_{F i x(c)},
$$

where the first map is defined in [II, (3.1.1) and (3.2.1)] using the standard identification $\mathcal{R} H o m(\mathcal{A}, \mathcal{B}) \cong \Delta^{!}(\mathbb{D} \mathcal{A} \otimes \mathcal{B})$, the second term is induced by the map $\mathbb{D} \mathcal{F} \otimes \mathcal{F} \rightarrow \Delta_{*} K_{X}$, adjoint to the evaluation map $\Delta^{*}(\mathbb{D} \mathcal{F} \otimes \mathcal{F})=\mathbb{D} \mathcal{F} \otimes \mathcal{F} \rightarrow K_{X}$, and the last one is the base change isomorphism $c^{!} \Delta_{*} \stackrel{\sim}{\rightarrow} \Delta_{*}^{\prime} c^{\prime !}$.

Since $H^{0}\left(C, \mathcal{R} H o m\left(c_{1}^{*} \mathcal{F}, c_{2}^{!} \mathcal{F}\right)\right)=\operatorname{Hom}\left(c_{1}^{*} \mathcal{F}, c_{2}^{!} \mathcal{F}\right) \cong \operatorname{Hom}\left(c_{2 !} c_{1}^{*} \mathcal{F}, \mathcal{F}\right)$, the map $H^{0}(C, \underline{\mathcal{I} r})$ gives rise to a map

$$
\mathcal{T} r=\mathcal{T} r_{c}: \operatorname{Hom}\left(c_{2 !} c_{1}^{*} \mathcal{F}, \mathcal{F}\right) \rightarrow H^{0}\left(C, \Delta_{*}^{\prime} K_{F i x(c)}\right)=H^{0}\left(F i x(c), K_{F i x(c)}\right),
$$

which we call the trace map.

b) For an open subset $\beta$ of $F i x(c)$, we denote by

$$
\mathcal{T} r_{\beta}: \operatorname{Hom}\left(c_{2 !} c_{1}^{*} \mathcal{F}, \mathcal{F}\right) \rightarrow H^{0}\left(\beta, K_{\beta}\right)
$$

the composition of $\mathcal{T} r$ and the restriction map $H^{0}\left(F i x(c), K_{F i x(c)}\right) \rightarrow H^{0}\left(\beta, K_{\beta}\right)$.

If, moreover, $\beta$ is proper over $k$, we denote by $L T_{\beta}: \operatorname{Hom}\left(c_{2 !} c_{1}^{*} \mathcal{F}, \mathcal{F}\right) \rightarrow \Lambda$ the composition of $\mathcal{T} r_{\beta}$ and the integration map $H^{0}\left(\beta, K_{\beta}\right) \rightarrow \Lambda$.

If $\beta$ is a connected component of Fix (c) which is proper over $k$, then $L T_{\beta}(u)$ is usually called the local term of $u$ at $\beta$.

2.2.2. Example. If $c=c_{t r}$ (see 2.1.3), then $H^{0}\left(\right.$ Fix $\left.(c), K_{\text {Fix }(c)}\right)=\Lambda$, each $\mathcal{F}$ is just a bounded complex of finitely generated free $\Lambda$-modules (modulo homotopy), and the trace map $\mathcal{T} r_{c_{t r}}$ coincides with the usual trace map $\operatorname{Hom}(\mathcal{F}, \mathcal{F}) \rightarrow \Lambda$.

2.2.3. Remark. Our trace map is equivalent to the map $\langle\cdot, \mathrm{Id}\rangle$, where

$$
\langle\cdot, \cdot\rangle: \operatorname{Hom}\left(c_{2 !} c_{1}^{*} \mathcal{F}, \mathcal{F}\right) \otimes \operatorname{Hom}(\mathcal{F}, \mathcal{F}) \rightarrow H^{0}\left(\beta, K_{\beta}\right)
$$

is the pairing, associated by Illusie [Il, (4.2.5)], to a pair of correspondences $c: C \rightarrow$ $X \times X$ and $\Delta: X \rightarrow X \times X$. However, our notion is more elementary.

As in [Il, Cor. 4.5], the trace maps commute with proper push-forwards.

2.2.4. Proposition. Let $[f]=\left(f, f^{\natural}, f\right)$ be a morphism from a correspondence $c: C \rightarrow X \times X$ to $b: B \rightarrow Y \times Y$ such that $f$ and $f^{\natural}$ are proper. Then the 
morphism $f^{\prime}:$ Fix $(c) \rightarrow F i x(b)$ induced by $[f]$ is proper as well, and for every c-morphism $u: c_{2 !} ! c_{1}^{*} \mathcal{F} \rightarrow \mathcal{F}$, we have an equality

$$
\mathcal{T} r_{b}\left([f]_{!}(u)\right)=f_{!}^{\prime}\left(\mathcal{T} r_{c}(u)\right) \in H^{0}\left(F i x(b), K_{F i x(b)}\right) .
$$

Applying the proposition to the case when $X$ and $C$ are proper over $k$ and $[f]$ is the structure morphism $[\pi]_{c}$ of 2.1 .3 , we deduce the well-known Lefschetz-Verdier trace formula (II, Cor. 4.7]).

2.2.5. Corollary. Let $c: C \rightarrow X \times X$ be a correspondence such that $C$ and $X$ are proper over $k$. Then for every $c$-morphism $u: c_{2} ! c_{1}^{*} \mathcal{F} \rightarrow \mathcal{F}$, we have an equality

$$
\operatorname{Tr}\left(R \Gamma_{c}(u)\right)=\sum_{\beta \in \pi_{0}(F i x(c))} L T_{\beta}(u) .
$$

\subsection{Invariant subsets.}

2.3.1. Definition. Let $c: C \rightarrow X \times X$ be a correspondence, and $Z \subset X$ a closed subset.

a) We say that $Z$ is $c$-invariant if $c_{1}\left(c_{2}^{-1}(Z)\right)$ is set-theoretically contained in $Z$.

b) We say that $Z$ is $c$-invariant in a neighborhood of fixed points if there exists an open neighborhood $W \subset C$ of $F i x(c)$ such that $Z$ is $\left.c\right|_{W}$-invariant, where $\left.c\right|_{W}$ : $W \rightarrow X \times X$ is the restriction of $c$.

2.3.2. Lemma. Let $c: C \rightarrow X \times X$ be a correspondence, and $Z \subset X$ a closed subset.

a) The set $W(Z):=C \backslash\left[\overline{c_{2}^{-1}(Z) \backslash c_{1}^{-1}(Z)}\right]$ is the largest open subset $W \subset C$ such that $Z$ is $\left.c\right|_{W}$-invariant, where by ${ }^{-1}$ we denote the closure.

b) $Z$ is locally c-invariant if and only if for each irreducible component $S$ of $c_{2}^{-1}(Z) \backslash c_{1}^{-1}(Z)$, the closures of $c_{1}(S)$ and $c_{2}(S)$ in $X$ do not intersect.

c) If $Z$ is locally c-invariant, then $Z$ is c-invariant in a neighborhood of fixed points.

Proof. a) and b) follow from definitions, and c) follows from a) and b).

2.3.3. Example. If $c_{2}$ is quasi-finite, then every closed point $x \in X$ is locally $c$-invariant. Indeed, $U:=X \backslash\left[c_{1}\left(c_{2}^{-1}(x)\right) \backslash x\right]$ is the required open neighborhood. As a result, every closed point $x \in X$ is locally $c$-invariant in a neighborhood of fixed points.

2.3.4. Definition. Let $c: C \rightarrow X \times X$ be a correspondence. We say that a correspondence $\bar{c}: \bar{C} \rightarrow \bar{X} \times \bar{X}$ is a compactification of $c$ if $\bar{X}$ and $\bar{C}$ are proper over $k, C \subset \bar{C}$ and $X \subset \bar{X}$ are open subsets, and $c$ is the restriction of $\bar{c}$.

The following lemma can be deduced from Lemma 2.3.2 b).

2.3.5. Lemma. Let $c: C \rightarrow X \times X$ be a correspondence and $U \subset X$ an open subset such that $c_{1}^{-1}(U)$ is dense in $C,\left.c_{1}\right|_{c_{1}^{-1}(U)}$ is proper, and $X \backslash U$ is locally c-invariant. Then there exists a compactification $\bar{c}: \bar{C} \rightarrow \bar{X} \times \bar{X}$ of c such that $\bar{X} \backslash U$ is locally $\bar{c}$-invariant.

2.3.6. Restriction of correspondences. Let $c: C \rightarrow X \times X$ be a correspondence, $u: c_{2 !} c_{1}^{*} \mathcal{F} \rightarrow \mathcal{F}$ a $c$-morphism, and $Z \subset X$ a closed subset.

a) If $Z$ is $c$-invariant, then $c$ induces a correspondence $\left.c\right|_{Z}: c_{2}^{-1}(Z)_{\text {red }} \rightarrow Z \times Z$, hence by 1.1.3. $u$ restricts to a $\left.c\right|_{Z}$-morphism $\left.u\right|_{Z}$. 
b) In general, let $W=W(Z) \subset C$ be as in Lemma 2.3 .2 a). Then $Z$ is $\left.c\right|_{W^{-}}$ invariant, and we denote the correspondence $\left.\left(\left.c\right|_{W}\right)\right|_{Z}$ defined in a) simply by $\left.c\right|_{Z}$. Moreover, by 1.1.3 $u$ restricts to a $\left.c\right|_{W}$-morphism $\left.u\right|_{W}$, hence by a) to a $\left.c\right|_{Z^{-}}$ morphism $\left.u\right|_{Z}:=\left.\left(\left.u\right|_{W}\right)\right|_{Z}$.

2.3.7. Example. If $c_{2}$ is quasi-finite, and $Z$ is a closed point $x$, then $\left.c\right|_{Z}=\left.c\right|_{x}$ is the correspondence $c_{1}^{-1}(x) \cap c_{2}^{-1}(x) \rightarrow\{x\} \times\{x\}$. Moreover, for each $y \in$ $c_{1}^{-1}(x) \cap c_{2}^{-1}(x)$, the restriction of $\left.u\right|_{x}$ to $\{y\} \rightarrow\{x\} \times\{x\}$ equals the endomorphism $u_{y}: \mathcal{F}_{x} \rightarrow \mathcal{F}_{x}$ defined in 1.1.7. Using 2.2.2 we see that $L T_{y}\left(\left.u\right|_{x}\right)=\operatorname{Tr}\left(u_{y}\right)$.

\subsection{Contracting correspondences.}

2.4.1. Definition. Let $c: C \rightarrow X \times X$ be a correspondence, and $Z \subset X$ a closed subscheme.

a) We say that $c$ stabilizes $Z$ if $c_{1}\left(c_{2}^{-1}(Z)\right)$ is scheme-theoretically contained in $Z$, i.e., if $c_{1}\left(\mathcal{I}_{Z}\right) \subset \dot{c_{2}}\left(\mathcal{I}_{Z}\right) \cdot \mathcal{O}_{C}$.

b) We say that $c$ is contracting near $Z$ if $c$ stabilizes $Z$ and there exists $n \in \mathbb{N}$ such that $\dot{c}\left(\mathcal{I}_{Z}\right)^{n} \subset \dot{c} \cdot\left(\mathcal{I}_{Z}\right)^{n+1} \cdot \mathcal{O}_{C}$.

c) We say that $c$ is contracting near $Z$ in a neighborhood of fixed points if there exists an open neighborhood $W \subset C$ of Fix $(c)$ such that $\left.c\right|_{W}: W \rightarrow X \times X$ is contracting near $Z$.

2.4.2. Remark. a) A geometric characterization of a contracting correspondence will be given in Remark 3.3 .5

b) If a correspondence $c$ is contracting near $Z$, then $c^{r i g}$ is contracting near $Z^{\text {rig }}$ in the sense of [Fu, Def. 3.1.1]. Furthermore, it is likely that the two notions are equivalent.

The proof of the following crucial result will occupy Section 3 .

2.4.3. Theorem. Let $c: C \rightarrow X \times X$ be a correspondence contracting near a closed subscheme $Z \subset X$ in a neighborhood of fixed points, and let $\beta$ be an open connected subset of Fix $(c)$ such that $c^{\prime}(\beta) \cap Z \neq \emptyset$. Then

a) $c^{\prime}(\beta)$ is contained in $Z$, hence $\beta$ is an open connected subset of Fix $\left(\left.c\right|_{Z}\right)$.

b) For every c-morphism $u: c_{2 !} c_{1}^{*} \mathcal{F} \rightarrow \mathcal{F}$, we have $\mathcal{T} r_{\beta}(u)=\mathcal{T} r_{\beta}\left(\left.u\right|_{Z}\right)$. In particular, if $\beta$ is proper, then $L T_{\beta}(u)=L T_{\beta}\left(\left.u\right|_{Z}\right)$.

To apply the result, we will use the following lemma.

2.4.4. Lemma. Let $c: C \rightarrow X \times X$ be a correspondence defined over $\mathbb{F}_{q}$.

a) Let $Z$ be a locally c-invariant closed subset of $X$ defined over $\mathbb{F}_{q}$. Then for each $n \in \mathbb{N}$ with $q^{n}>\operatorname{ram}\left(c_{2}, Z\right)$, the correspondence $c^{(n)}$ is contracting near $Z$ in a neighborhood of fixed points.

b) If $c_{2}$ is quasi-finite, then for each $q^{n}>\operatorname{ram}\left(c_{2}\right)$, the correspondence $c^{(n)}$ is contracting near every closed point $x$ of $X$ in a neighborhood of fixed points.

\subsection{Proof of Theorem 1.2.2.}

2.5.1. Proof of a). The assertion follows from Lemma $2.4 .4 \mathrm{~b}$ ), Theorem 2.4.3 a) and the fact that for every closed point $x$ of $X$, the set Fix $\left(\left.c\right|_{x}\right)$ is finite.

2.5.2. Proof of $\mathbf{c})$. By Corollary 2.2.5, we have an equality

$$
\operatorname{Tr}\left(R \Gamma_{c}(u)\right)=\sum_{\beta \in \pi_{0}\left(F i x\left(c^{(n)}\right)\right)} L T_{\beta}(u)
$$


Pick any $\beta \in \pi_{0}\left(F i x\left(c^{(n)}\right)\right)$ such that $c_{2}(\beta) \subset X \backslash U$. Since $q^{n}>\operatorname{ram}\left(c_{2}, X \backslash U\right)$, we conclude from Lemma 2.4.4 a) and Theorem 2.4.3 that $\beta$ is a connected component of Fix $\left(\left.c\right|_{X \backslash U} ^{(n)}\right)$, and $L T_{\beta}(u)$ equals $L T_{\beta}\left(\left.u\right|_{X \backslash U}\right)$. As $\left.\mathcal{F}\right|_{X \backslash U}=0$, we get that $L T_{\beta}\left(\left.u\right|_{X \backslash U}\right)=0$, hence also $L T_{\beta}(u)=0$.

Pick now any $\beta \in \pi_{0}\left(\operatorname{Fix}\left(c^{(n)}\right)\right)$ such that $c_{2}(\beta) \cap U \neq \emptyset$. As $q^{n}>\operatorname{ram}\left(\left.c_{2}\right|_{c_{2}^{-1}(U)}\right)$, such $\beta$ is simply a point $y \in \operatorname{Fix}\left(c^{(n)}\right) \cap c^{-1}(U)$ (by a)). Moreover, by Lemma 2.4.4 b), Theorem 2.4.3b) and 2.3.7, $L T_{\beta}(u)$ equals $L T_{y}\left(\left.u\right|_{x}\right)=\operatorname{Tr}\left(u_{y}\right)$. This shows that the right-hand side of (2.5) is equal to that of (1.1), as claimed.

2.5.3. Proof of $\mathbf{b})$. For the proof we can replace $c$ and $u$ by their restrictions to $c_{1}^{-1}(U)$. Then the assumptions of Lemma 2.3.5 are satisfied, hence there exists a compactification $\bar{c}: \bar{C} \rightarrow \bar{X} \times \bar{X}$ of $c$ such that $\bar{X} \backslash U$ is locally $\bar{c}$-invariant. We claim that $d:=\max \left\{\operatorname{ram}\left(\left.c_{2}\right|_{c_{2}^{-1}(U)}\right), \operatorname{ram}\left(\bar{c}_{2}, \bar{X} \backslash U\right)\right\}$ has the required property.

Let $c^{0}: C^{0}=c_{1}^{-1}(U) \rightarrow U \times X$ be the restriction of $c, u^{0}$ the restriction of $u$ to $c^{0}$, and $[j]=\left(j^{0}, j_{C^{0}}, j\right)$ the inclusion map of $c^{0}$ into $\bar{c}$. Then $[j]$ satisfies assumption (iii) of 2.1.2, and therefore $u^{0}$ extends to a $\bar{c}$-morphism $\bar{u}:=[j]_{!}\left(u^{0}\right): \bar{c}_{2 !} \bar{c}_{1}^{*} \overline{\mathcal{F}} \rightarrow \overline{\mathcal{F}}$, where $\overline{\mathcal{F}}:=j_{!}(\mathcal{F})=j_{!}^{0}\left(\left.\mathcal{F}\right|_{U}\right)$.

We claim that the equality (1.1) for $c, U$, and $u$ is equivalent to that for $\bar{c}, U$, and $\bar{u}$. Indeed, since $[\pi]_{c^{0}}=[\pi]_{\bar{c}} \circ[j]$, the equality $\operatorname{Tr}\left(R \Gamma_{c}(\bar{u})\right)=\operatorname{Tr}\left(R \Gamma_{c}(u)\right)$ follows from 2.1.3, while the equality $\operatorname{Tr}\left(\bar{u}_{y}\right)=\operatorname{Tr}\left(u_{y}\right)$ for all $y \in F i x\left(c^{(n)}\right) \cap c^{\prime-1}(U)$ is clear. Now the assertion follows from part $c)$ of the theorem proven above.

\section{LOCAL TERMS FOR CONTRACTING CORRESPONDENCES}

This section is devoted to the proof of Theorem 2.4.3.

\subsection{Additivity of the trace maps.}

3.1.1. Notation. Let $c: C \rightarrow X \times X$ be a correspondence, $u: c_{2} c_{1}^{*} \mathcal{F} \rightarrow \mathcal{F}$ a $c$-morphism, and $Z \subset X$ a $c$-invariant closed subset.

a) Let $\left.c\right|_{Z}$ and $\left.u\right|_{Z}$ be as in 2.3.6 a), and let $[i]_{Z}$ be the closed embedding of $\left.c\right|_{Z}$ into $c$. Then $[i]_{Z}$ satisfies assumption (ii) of 2.1.2, hence $u$ gives rise to a $c$-morphism $[i]_{Z !}\left(\left.u\right|_{Z}\right)$.

b) Put $U:=X \backslash Z$. Then $c_{1}^{-1}(U) \subset c_{2}^{-1}(U)$, and we denote by $\left.c\right|_{U}: c_{1}^{-1}(U) \rightarrow$ $U \times U$ the restriction of $c$, by $\left.u\right|_{U}$ the restriction of $u$ to $\left.c\right|_{U}$ (see 1.1.3), and by $[j]_{U}$ the open embedding of $\left.c\right|_{U}$ into $c$. Since $[j]_{U}$ satisfies assumption (i) of [2.1.2, $\left.u\right|_{U}$ extends to a $c$-morphism $[j]_{U !}\left(\left.u\right|_{U}\right)$.

As in [Pi, Prop. 2.4.3] and [II, 4.13], the trace maps are additive.

3.1.2. Proposition. In the notation of 3.1.1, we have an equality

$$
\mathcal{T} r_{c}(u)=\mathcal{T} r_{c}\left([i]_{Z !}\left(\left.u\right|_{Z}\right)\right)+\mathcal{T} r_{c}\left([j]_{U !}\left(\left.u\right|_{U}\right)\right) .
$$

\subsection{Specialization.}

3.2.1. Notation. For a scheme $X$ over $k$, set $X_{\mathbb{A}}:=X \times \mathbb{A}^{1}$. For a morphism $f: X \rightarrow Y$ of schemes over $k$, set $f_{\mathbb{A}}:=f \times \operatorname{Id}_{\mathbb{A}^{1}}: X_{\mathbb{A}} \rightarrow Y_{\mathbb{A}}$. For a scheme $\widetilde{X}$ over $\mathbb{A}^{1}$, we denote by $\widetilde{X}_{s}$ its fiber over $0 \in \mathbb{A}^{1}$, and by $\Psi_{\widetilde{X}}: D_{c t f}^{b}(\widetilde{X}, \Lambda) \rightarrow D_{c t f}^{b}\left(\widetilde{X}_{s}, \Lambda\right)$ the corresponding functor of nearby cycles. 


\subsubsection{Specialization functor.}

a) We say that a scheme $\widetilde{X}$ over $\mathbb{A}^{1}$ lifts a scheme $X$ over $k$ if it is equipped with a morphism $\varphi=\varphi_{X}: \widetilde{X} \rightarrow X$ such the corresponding morphism $\widetilde{X} \rightarrow X_{\mathbb{A}}=X \times \mathbb{A}^{1}$ is an isomorphism over $\mathbb{A}^{1} \backslash\{0\}$. In this case, we define a functor

$$
s p_{\widetilde{X}}:=\Psi_{\widetilde{X}} \circ \varphi^{*}: D_{c t f}^{b}(X, \Lambda) \rightarrow D_{c t f}^{b}\left(\widetilde{X}_{s}, \Lambda\right) .
$$

b) We say that a morphism $\tilde{f}: \tilde{X} \rightarrow \widetilde{Y}$ of schemes over $\mathbb{A}^{1}$ lifts a morphism $f: X \rightarrow Y$ of schemes over $k$ if $\widetilde{X}$ lifts $X, \widetilde{Y}$ lifts $Y$, and $\varphi_{Y} \circ \widetilde{f}=f \circ \varphi_{X}$. In this case, we have base change morphisms $B C^{*}: \widetilde{f}_{s}^{*} s p_{\widetilde{Y}} \rightarrow s p_{\widetilde{X}} f^{*}, B C_{*}: s p_{\widetilde{Y}} f_{*} \rightarrow \widetilde{f}_{s *} s p_{\widetilde{X}}$, $B C^{!}: s p_{\widetilde{X}} f^{!} \rightarrow \widetilde{f}_{s}^{!} s p_{\widetilde{Y}}$ and $B C_{!}: \widetilde{f}_{s !} s p_{\widetilde{X}} \rightarrow s p_{\widetilde{Y}} f_{!}$, induced by the corresponding base change morphisms for $\Psi$.

3.2.3. Examples. a) If $\widetilde{X}=X_{\mathbb{A}}$ and $\varphi$ is the projection map, then $\widetilde{X}_{s}=X$, and the functor $s p_{\widetilde{X}}$ is isomorphic to the identity functor.

b) If $f: X \rightarrow$ Spec $k$ and $\widetilde{f}: \widetilde{X} \rightarrow \mathbb{A}^{1}$ are the structure morphisms, then the composition $B C^{!} \circ B C_{*}: f_{*} f^{!} \Lambda=s p_{\mathbb{A}^{1}} f_{*} f^{!} \Lambda \rightarrow \widetilde{f}_{s *} \widetilde{f}_{s}^{!} s p_{\mathbb{A}^{1}} \Lambda=\widetilde{f}_{s *} \widetilde{f}_{s}^{!} \Lambda$ defines a morphism $H^{0}\left(X, K_{X}\right) \rightarrow H^{0}\left(\widetilde{X}_{s}, K_{\widetilde{X}_{s}}\right)$, which we will denote simply by $s p_{\widetilde{X}}$.

3.2.4. Specialization of correspondences. Let $c: C \rightarrow X \times X$ be a correspondence, $u: c_{2 !} c_{1}^{*} \mathcal{F} \rightarrow \mathcal{F}$ a $c$-morphism, $\widetilde{c}: \widetilde{C} \rightarrow \widetilde{X} \times \widetilde{X}$ a correspondence over $\mathbb{A}^{1}$ lifting $c$, and $\widetilde{c}_{s}$ the fiber of $\widetilde{c}$ over $0 \in \mathbb{A}^{1}$. Then $u$ gives rise to a $\widetilde{c}_{s}$-morphism

$$
s p_{\widetilde{c}}(u): \widetilde{c}_{s 2 !} \widetilde{c}_{s 1}^{*} s p_{\widetilde{X}} \mathcal{F} \stackrel{B C^{*}}{\longrightarrow} \widetilde{c}_{s 2 !} s p_{\widetilde{C}} c_{1}^{*} \mathcal{F} \stackrel{B C_{1}}{\longrightarrow} s p_{\widetilde{X}} c_{2 !} c_{1}^{*} \mathcal{F} \stackrel{u}{\longrightarrow} s p_{\widetilde{X}} \mathcal{F} .
$$

As in [Fu, Prop. 1.7.1], the trace maps commute with the specialization.

3.2.5. Proposition. In the notation of 3.2.4, assume that $\widetilde{c}$ extends to a lifting of some compactification $\bar{c}: \bar{C} \rightarrow \bar{X} \times \bar{X}$ of $c$. Then we have an equality

$$
\mathcal{T} r_{\widetilde{c}_{s}}\left(s p_{\widetilde{c}}(u)\right)=s p_{F i x(\widetilde{c})}\left(\mathcal{T} r_{c}(u)\right) \in H^{0}\left(\operatorname{Fix}\left(\widetilde{c}_{s}\right), K_{F i x\left(\widetilde{c}_{s}\right)}\right) .
$$

3.3. Deformation to the normal cone. We will apply the specialization in the following particular case.

3.3.1. Notation. Let $X$ be a scheme over $k$, and $Z \subset X$ a closed subscheme.

a) Denote by $\widetilde{X}_{Z}$ the spectrum of the $\mathcal{O}_{X}$-subalgebra $\mathcal{O}_{X}\left[t, \frac{\mathcal{I}_{Z}}{t}\right] \subset \mathcal{O}_{X}\left[t, t^{-1}\right]$. The embedding $\mathcal{O}_{X}[t] \hookrightarrow \mathcal{O}_{X}\left[t, \frac{\mathcal{I}_{Z}}{t}\right]$ gives rise to the birational projection $\widetilde{\varphi}: \widetilde{X}_{Z} \rightarrow$ $X_{\mathbb{A}}$, which is an isomorphism over $\mathbb{A}^{1} \backslash\{0\}$.

b) The special fiber $\left(\widetilde{X}_{Z}\right)_{s}=\mathcal{S} p e c\left(\bigoplus_{n=0}^{\infty}\left(\mathcal{I}_{Z}\right)^{n} /\left(\mathcal{I}_{Z}\right)^{n+1}\right)$ is the normal cone of $X$ to $Z$, which we denote by $N_{Z}(X)$.

c) The projection $\mathcal{O}_{X}\left[t, \frac{\mathcal{I}_{Z}}{t}\right] \rightarrow\left(\mathcal{O}_{X} / \mathcal{I}_{Z}\right)[t]=\mathcal{O}_{Z}[t]$ defines a closed embedding $\widetilde{\iota}: Z_{\mathbb{A}} \hookrightarrow \widetilde{X}_{Z}$. The special fiber $\widetilde{\iota}_{s}: Z \hookrightarrow N_{Z}(X)$ identifies $Z$ with the zero section of $N_{Z}(X)$.

d) Since $s p_{Z_{\mathrm{A}}}$ is the identity functor (see 3.2.3), the map $B C^{*}$ (from 3.2.2) for the embedding $\widetilde{\iota}$ from $\mathrm{c}$ ) defines a morphism

$$
\left.\left.s p_{\widetilde{X}_{Z}}(\mathcal{F})\right|_{Z} \rightarrow \mathcal{F}\right|_{Z} \text {. }
$$

The following property of the deformation to the normal cone, proven in $\mathrm{Ve}, \S 8$, (SP5)], is crucial for the whole proof.

\subsubsection{Lemma. The morphism (3.2) is an isomorphism.}

The following lemma is straightforward. 
3.3.3. Lemma. Let $f: X_{1} \rightarrow X_{2}$ be a morphism of schemes over $k, Z_{2} \subset X_{2}$ a closed subscheme, and $Z_{1}$ a closed subscheme of $f^{-1}\left(Z_{2}\right)$, that is, $f^{\cdot}\left(\mathcal{I}_{Z_{2}}\right) \subset \mathcal{I}_{Z_{1}}$.

a) The morphism $f_{\mathbb{A}}: X_{1 \mathbb{A}} \rightarrow X_{2 \mathbb{A}}$ lifts uniquely to the map $\widetilde{f}:\left(\widetilde{X}_{1}\right)_{Z_{1}} \rightarrow\left(\widetilde{X}_{2}\right)_{Z_{2}}$.

b) The image $\widetilde{f}_{s}\left(N_{Z_{1}}\left(X_{1}\right)\right)$ is supported set-theoretically at the zero section of $N_{Z_{2}}\left(X_{2}\right)$ if and only if there exists $n \in \mathbb{N}$ such that $f^{\cdot}\left(\mathcal{I}_{Z_{2}}\right)^{n} \subset\left(\mathcal{I}_{Z_{1}}\right)^{n+1}$.

3.3.4. Deformation of correspondences. Let $c: C \rightarrow X \times X$ be a correspondence, and $Z \subset X$ a closed subscheme. Then by Lemma 3.3.3, $c$ lifts to a correspondence $\widetilde{c}_{Z}: \widetilde{C}_{c^{-1}(Z \times Z)} \rightarrow \widetilde{X}_{Z} \times \widetilde{X}_{Z}$. Moreover, if $\bar{c}: \bar{C} \rightarrow \bar{X} \times \bar{X}$ is a compactification of $c$, and $\bar{Z} \subset \bar{X}$ is the closure of $Z$, then the correspondence $\widetilde{\bar{c}}_{\bar{Z}}$ extends $\widetilde{c}_{Z}$ and lifts $\bar{c}$. In particular, Proposition 3.2 .5 holds for $c$ and $\widetilde{c}_{Z}$.

3.3.5. Remark. Recall that a correspondence $c: C \rightarrow X \times X$ stabilizes a closed subscheme $Z \subset X$ if and only if $c^{-1}(Z \times Z)=c_{2}^{-1}(Z)$. Therefore, by Lemma 3.3.3 b), $c$ is contracting near $Z$ if and only if $c$ stabilizes $Z$ and the image of $\left(\widetilde{c}_{Z}\right)_{1 s}$ is supported set-theoretically at the zero section $Z \subset N_{Z}(X)$.

3.4. Proof of Theorem 2.4.3. Choose an open neighborhood $W \subset C$ of Fix(c) such that $\left.c\right|_{W}$ is contracting near $Z$. Replacing $c$ by $\left.c\right|_{W}$, we can assume that $c$ is contracting near $Z$. Moreover, replacing further $C$ by an open subset $C \backslash[F i x(c) \backslash \beta]$ we can assume that $\operatorname{Fix}(c)=\beta$, hence $\mathcal{T} r_{\beta}=\mathcal{T} r_{c}$. For the proof we apply the construction of 3.3.4.

3.4.1. Proof of a). By Remark 3.3.5, the image of $\left(\widetilde{c}_{Z}\right)_{1 s}$ is supported at $Z \subset$ $N_{Z}(X)$. Since $\left.\widetilde{F i x(c)}\right)_{c^{\prime-1}(Z)}$ is a closed subscheme of Fix $\left(\widetilde{c}_{Z}\right)$, the image of $\widetilde{c}_{s}^{\prime}$ : $N_{c^{\prime-1}(Z)}(\beta) \rightarrow N_{Z}(X)$ is then supported at $Z$ as well. Using the "only if" statement of Lemma $3.3 .3 \mathrm{~b}$ ) we conclude that $\beta_{\text {red }}=\left(c^{\prime}\right)^{-1}(Z)_{\text {red }}$, as claimed.

3.4.2. Proof of b). Put $U:=X \backslash Z$. Then we have $\mathcal{T} r_{c}(u)=\mathcal{T} r_{c}\left([i]_{Z !}\left(\left.u\right|_{Z}\right)\right)+$ $\mathcal{T} r_{c}\left([j]_{U !}\left(\left.u\right|_{U}\right)\right.$ ) (by Proposition 3.1.2) and $\mathcal{T} r_{c}\left([i]_{Z !}\left(\left.u\right|_{Z}\right)\right)=i_{Z !}^{\prime} \mathcal{T} r_{\left.c\right|_{Z}}\left(\left.u\right|_{Z}\right)$ (by Proposition 2.2.4). Since $i_{Z !}^{\prime}$ is the identity map (by a)), it remains to show that $\mathcal{T} r_{c}\left([j]_{U !}\left(\left.u\right|_{U}\right)\right)=0$. For this, we may replace $\mathcal{F}$ by $j !\left(\left.\mathcal{F}\right|_{U}\right)$ and $u$ by $[j]_{U !}\left(\left.u\right|_{U}\right)$. In this case $\left.\mathcal{F}\right|_{Z}=0$, and we claim that $\mathcal{T} r_{c}(u)=0$.

By Proposition 3.2.5 for correspondences $c$ and $\widetilde{c}_{Z}$, it will suffice to show that

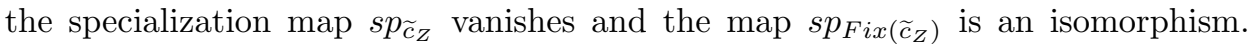
Since the image of $\left(\widetilde{c}_{Z}\right)_{1 s}$ is supported at $Z \subset N_{Z}(X)$, while $\left.\left.s p_{\widetilde{X}_{Z}}(\mathcal{F})\right|_{Z} \cong \mathcal{F}\right|_{Z}=0$ (by Lemma 3.3.2), the sheaf $\left(\widetilde{c}_{Z}\right)_{1 s}^{*} s p_{\widetilde{X}_{Z}}(\mathcal{F})$ vanishes. This implies the vanishing of $s p_{\widetilde{c}_{Z}}$. On the other hand, since $\left(\widetilde{c}_{Z}\right)_{2 s}^{-1}(Z)$ equals $c_{2}^{-1}(Z) \subset N_{c_{2}^{-1}(Z)}(C)$, we see that Fix $\left(\widetilde{c}_{Z}\right)_{\text {red }}=\left(\beta_{\mathcal{D}}\right)_{\text {red }} \subset c_{2}^{-1}(Z)_{\mathcal{D}}$. Thus $s p_{\text {Fix }\left(\widetilde{c}_{Z}\right)}$ is an isomorphism by 3.2 .3 ,

This completes the proof of Theorem 2.4.3 and hence also of Theorem 1.2 .2

\section{REFERENCES}

[Fu] K. Fujiwara, Rigid geometry, Lefschetz-Verdier trace formula and Deligne's conjecture, Invent. Math. 127 (1997), no. 3, 489-533. MR1431137 (99c:14030)

[KV] D. Kazhdan and Y. Varshavsky, On the cohomology of the moduli spaces of F-bundles: stable cuspidal Deligne-Lusztig part, in preparation.

[Il] L. Illusie, Formule de Lefschetz, in Coholologie $\ell$-adique et fonctions L, SGA5, Lecture Notes in Mathematics 589, Springer-Verlag, 1977, pp. 73-137. MR0491704 (58:10907) 
[Pi] R. Pink, On the calculation of local terms in the Lefschetz-Verdier trace formula and its application to a conjecture of Deligne, Ann. of Math. 135 (1992), no. 3, 483-525. MR.1166642 (93m:14017)

[Ve] J.-L. Verdier, Spécialisation de faisceaux et monodromie modérée, in Analysis and topology on singular spaces, Astérisque 101-102, 1983, pp. 332-364. MR0737938 (86f:32010)

Institute of Mathematics, Hebrew University, Givat-Ram, Jerusalem 91904, Israel

E-mail address: vyakov@math.huji.ac.il 